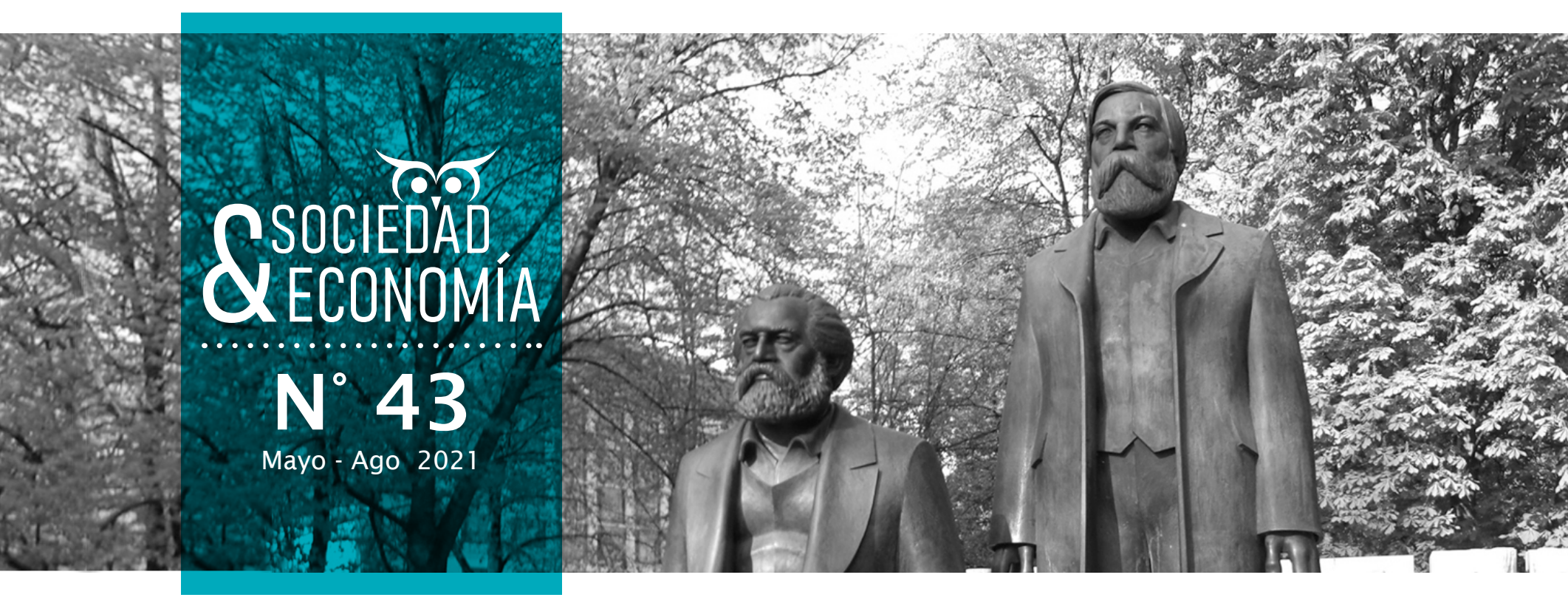

\title{
Algunas consideraciones sobre aspectos conceptuales en Historia del Pensamiento Económico
}

Some Considerations on Conceptual Issues in History of Economic Thought

Germán Raúl Chaparro'

Universidad Nacional de Colombia, Bogotá, Colombia; Universidad Central, Bogotá, Colombia.

시가.chaparrog@unal.edu.co

(D) https://orcid.org/0000-0002-6443-0021

Recibido: 23-07-2020

Aceptado: 15-10-2020

Publicado: 01-05-2021

1 Magíster en Economía. 


\title{
Resumen
}

El objetivo de este artículo es reivindicar la importancia de la enseñanza y práctica de la Historia del Pensamiento Económico, en tanto medio que ofrece una visión general e íntegra del campo de la economía que favorece el avance de la teoría económica. Para esto, se propone recuperar el método de la matriz conceptual. Se concluye que esta alternativa tiene el potencial de contribuir a desarrollar habilidades cognitivas de alto nivel y pensamiento crítico.

Palabras clave: enseñanza de la economía; historia del pensamiento económico; historia conceptual; método de la matriz.

Clasificación JEL: B40; N01.

\begin{abstract}
This paper aims to vindicate the importance of the teaching and practice of the History of Economic Thought, as a medium that offers a general and integrated view of the field of Economics that favors the advancement of economic theory. To do so, it is proposed to recover the matrix method. It is concluded that this alternative has the potential to contribute to the development of high-level cognitive skills and critical thinking.

Keywords: Teaching of economics; history of economic thought; conceptual history; matrix method.

JEL Classification: B40; N01.

\section{Financiación}

Este artículo es un producto del proyecto de investigación: "La enseñanza de la economía política en América Latina", avalado por el Grupo de investigación de Protección Social de la Universidad Nacional de Colombia (sede Bogotá). Una versión preliminar del artículo fue presentada en la IV Escuela de Verano CONCEPTA "Iberoamérica en perspectiva global", que se llevó a cabo entre el 15 y el 26 de julio de 2019 en El Colegio de México.
\end{abstract}

\section{Conflicto de interés}

El autor declara no tener ningún conflicto de interés en la publicación de este artículo. 
Sociedad y Economía N 43 (Mayo - Ago 2021) / e-ISSN: 2389-9050 / e10610458

Algunas consideraciones sobre aspectos conceptuales en Historia del Pensamiento Económico

\section{Introducción}

Este artículo tiene por objeto reivindicar la importancia del estudio y la práctica de la Historia del Pensamiento Económico (HPE). Específicamente, se realiza por la visión de conjunto que ofrece, por el sentido de dirección y lenguaje común que previene el riesgo de disgregación de la disciplina en especialidades, y por la contribución en el fomento del trabajo interdisciplinar y el avance de la teoría económica. Claramente, la comprensión de la realidad económica y la construcción de la teoría misma no pueden evadir ni la referencia a las grandes preguntas que unifican el campo de estudio, ni la referencia a la etapa de conceptualización que precede a la formulación de proposiciones y la construcción de modelos. En tal sentido, por considerar que ambos referentes son objetos de estudio privilegiados por la HPE, se propone como hipótesis la necesidad de recuperar los conceptos como objeto de análisis crítico. Esto es, identificar e interpretar los presupuestos conceptuales sobre los que se funda cada sistema de pensamiento en su intención de dar respuesta a las grandes preguntas en contextos históricos determinados. En tal sentido, se sugiere que el uso de las metodologías de la nueva historia política, en particular de la Ilamada Escuela de Cambridge $y$ de la Begriffsgeschichte (historia conceptual alemana), ofrece a estudiantes e investigadores una caja de herramientas valiosa en la tarea de mejorar la comprensión del campo de estudio, al permitir reconocer la historicidad de los conceptos y de los dispositivos argumentativos que subyacen a un modo de producción de pensamiento. La metodología en mención resulta potencialmente valiosa para promover el desarrollo de habilidades cognitivas de alto nivel, un pensamiento elevado en términos de complejidad y una actitud crítica para comparar y evaluar las distintas teorías económicas².

Una aproximación histórica a los conceptos supone intentar entender el pasado en sus pro-

2 Recuérdese que Keynes (1936/2013) propone que el objetivo del análisis económico "no es proporcionar una máquina o método de manipulación ciega [...] sino un método de pensamiento organizado y ordenado sobre problemas determinados" (p. 297). pios términos y evitar que las interpretaciones de la historia sean distorsionadas por la utilización de categorías presentes para entender situaciones del pasado. Frente a lo último, se evita usar categorías que no se corresponden con el uso lingüístico en el pasado o con las prácticas reales registradas por los conceptos entonces usados. En tal sentido, una descripción razonable de los tiempos pasados demanda exactitud filológica, de suerte que la terminología usada en un contexto particular sea derivada de las fuentes mismas, con el objetivo de evitar incurrir en anacronismos conceptuales. En otras palabras, evitar atribuir a los autores palabras, conceptos o significados que ellos no utilizaron y que podrían no pertenecer al plano de posibilidades de enunciación que estaban a su disposición. En el contexto de la HPE, la semántica histórica proporciona rigor a la definición de los conceptos y, por lo tanto, a los bloques constructivos de mayor escala, como proposiciones, teorías y modelos, lo que implica reconocer que estos están históricamente determinados.

La investigación en historia de los conceptos es compatible con el estudio de las grandes temáticas unificadoras de la teoría económica; por ejemplo, las cuestiones sobre la coordinación humana y el funcionamiento de los mercados, o la explicación de fenómenos como las instituciones y el cambio institucional. Ciertamente, las grandes teorías no son un mero conjunto de "ideas", entendidas como unidades de análisis que recogen un significado esencial que migra en el tiempo y se adap$\tan$ a cualquier circunstancia. Antes bien, éstas suelen haber sido formuladas bajo una serie de presupuestos analíticos históricamente definidos; en este sentido, son una forma particular de lenguaje articulado del cual se derivan facultades cognitivas y que revelan formas de percibir, describir y construir la realidad económica. Tratar de acceder a las condiciones de posibilidad de enunciación de un discurso implica trascender el plano de los significados y procurar entrar en su dimensión performativa, preguntar por los usos del lenguaje y entender cómo un autor pudo decir lo que dijo y por qué lo dijo. Para hacerlo, la historia de los 
conceptos identifica y rastrea históricamente los conceptos, recurre a análisis diacrónicos y sincrónicos para identificar tanto similitudes y consensos como diferencias y contradicciones al interior de la obra de un autor o escuela de pensamiento, o entre autores o escuelas. Esta estrategia puede contribuir a facilitar la comprensión de la forma en que evoluciona la teoría económica.

En el contexto de la enseñanza de la HPE, a nivel de pregrado, Peterson y Bean (1998) proponen una matriz conceptual como método guía para ayudar a los estudiantes a organizar y sistematizar los contenidos temáticos del curso, por autor o escuela y según conceptos o temas. Los autores aseguran que tal estrategia permite interconectar contenidos y contribuye a generar pensamiento crítico de más alto nivel. Al respecto, Ekelund y Hébert (1998) critican la propuesta, ponen en duda su utilidad y señalan aspectos que la hacen inconveniente. Empero, en el presente trabajo se sugiere que la propuesta de Peterson y Bean (1998) no se debe descartar. Para el particular, la matriz conceptual formulada es una alternativa didáctica, la cual, complementada con las advertencias metodológicas de la historia de los conceptos, puede contribuir de manera significativa a alcanzar los objetivos. Específicamente, desarrollar las capacidades cognitivas de alto nivel, y estrechar los vínculos de la historia del pensamiento con la historia de los hechos económicos y el trabajo en teoría económica propiamente dicho. En definitiva, contribuye a mejorar los procesos de enseñanza-aprendizaje.

El presente artículo está conformado por seis secciones. Entonces, además de esta introducción, el documento se organiza de la siguiente manera. En la segunda sección, se reivindica la importancia del estudio, la enseñanza y la investigación en la HPE, lo que implica hacer un balance de su estado actual; en la tercera, se presenta la propuesta de matriz conceptual elaborada por Peterson y Bean (1998); por su parte, la cuarta describe la experiencia en el uso de matriz conceptual; en la quinta, se señalan algunas advertencias metodológicas hechas por Skinner (1969/2007) que se conside- ran relevantes para potenciar el método de la matriz. Por último, la sexta sección presenta las conclusiones.

\section{Importancia de la Historia del Pensamiento Económico}

La investigación y la enseñanza de la HPE está en crisis. En los últimos treinta años, el desarrollo de la ciencia económica se ha caracterizado por una creciente especialización (Trautwein, 2017; Davis, 2019), orientada a enfrentar problemas cada vez más delimitados y con el recurso metodológico a experimentos naturales, ensayos controlados aleatorios y técnicas empíricas, que en general privilegian la técnica y los datos sobre la teoría y la interpretación (Angrist y Pischke, 2010). La creciente parcelación de la economía en subdisciplinas, esto es, en compartimentos cada vez más específicos como la economía laboral, de la salud, del sector público, del bienestar, ambiental, del comportamiento y de la organización industrial, es un fenómeno que implica un doble proceso de especialización y expansión del campo de estudio. La especialización es necesaria para profundizar el conocimiento científico de campos específicos y, además, tiene el potencial de permitir el desarrollo de conocimientos, herramientas e instrumentos que podrían estar disponibles o tener aplicación en otros campos. Por su parte, el progresivo ensanchamiento del campo de estudio es un proceso de extensión de la frontera en que incursionan los economistas con su caja de herramientas, lo que tiene el potencial de generar instancias de trabajo colaborativo con profesionales de otras disciplinas. De esta manera, la ciencia económica contemporánea se caracteriza por el estudio de teorías especializadas aplicadas a mercados específicos.

Sin embargo, el doble proceso de división y expansión del campo de estudio no está libre de riesgos: implica el peligro de balcanización, de generar una tendencia al desarrollo de la investigación económica en compartimentos estancos, con poca o nula comunicación y articulación entre los elementos, es decir, entre las subdisciplinas. El riesgo es evidente si se 
tiene en cuenta que estudiantes e investigadores se interesan cada vez menos en las visiones globales o generalistas de la economía (Trautwein, 2017). Es decir, los enfoques que tradicionalmente se han ocupado de las cuestiones fundamentales de la ciencia económica, como el problema de la coordinación social o las teorías acerca del funcionamiento de los mercados y del sistema capitalista, retos propios de las grandes escuelas de pensamiento económico, en particular de las teorías clásica, neoclásica, marxista y keynesiana. En este sentido, Rebeca Gómez y Alexander Tobón (2009, p. 244) han interpretado que, mientras la teoría económica contemporánea se asocia con el estudio de las teorías especializadas, se ha reservado para la HPE el estudio de los paradigmas y las teorías generales. Las primeras se asocian a la frontera teórica y la aplicación de modelos, en tanto que los segundos se asimilan a visiones retrospectivas.

El progresivo desinterés por la HPE dentro de la Economía empezó a evidenciarse en los años sesenta del siglo XX (Gordon, 1965; Goodwin, 2008) y, en las últimas décadas, es un hecho reconocido por profesores de la disciplina alrededor del mundo (Cardoso, 1995). En la práctica, esto se expresa en la reducción del número de cursos que sobre estas temáticas es ofrecido en los programas profesionales de Economía o del número de artículos en el área de HPE publicados en las revistas de Economía (García y Giraud, 2016). La situación revela que la HPE tiene dificultades para encontrar su posición dentro de la disciplina, incluso para alcanzar el estatus de subdisciplina dentro de la teoría económica moderna. De hecho, parece alejarse de la Economía y acercarse más a la Historia, en particular a la Historia Intelectual o a la Historia de las Ideas, o a la Filosofía de la Ciencia. Mark Blaug (2001) expresó en una sentencia lapidaria lo que parece ser la mentalidad de los economistas modernos: ¡Sin Historia de las Ideas, por favor, somos economistas!

Esta pérdida de interés es atribuible, en parte, a la consolidación hegemónica de una vertiente del pensamiento como paradigma dominante, la escuela neoclásica, y, también, a los entornos institucionales de la educación y la investigación. Dentro de la vertiente principal de la teoría económica predomina una visión acumulativa y absolutista del conocimiento, de reconstrucciones racionales o de historia whig (Samuelson, 1987). Es decir, en general, una mirada anacrónica sobre el pasado y una concepción absolutista del conocimiento según la cual el corpus de la teoría económica dominante en el presente ha incorporado todas las ideas y enseñanzas relevantes de las teorías del pasado. Así, dentro del mainstream se concibe el estudio y la práctica de la HPE como un ejercicio sospechoso de mera curiosidad intelectual, que no contribuye a generar conocimiento teórico y que, en consecuencia, implica un costo de oportunidad elevado que distrae e inhibe la creatividad para trabajar en la frontera del conocimiento.

Un prejuicio común consiste en identificar la HPE con una trinchera de la heterodoxia, desde la cual se lanzan ataques, críticas externas, a la corriente dominante. De hecho, el sistema de clasificación desarrollado por el Journal of Economic Literature (JEL), ampliamente utilizado para catalogar la literatura académica en el campo de la economía, agrupa dentro de una misma categoría general, la categoría B, los trabajos en "Historia del Pensamiento Económico, Metodología y Enfoques Heterodoxos".

Por otro lado, existen barreras institucionales, como por ejemplo la tendencia a la reducción de la duración de los programas de pregrado. En este caso, de los programas de economía (Castro y Raffo, 2016, p. 161), que en su organización, dada la escasez de tiempo, deciden dar prioridad a los campos de economía aplicada, dejando al margen los cursos de carácter teórico e histórico, los cuales se considera que representan un costo de oportunidad alto para los estudiantes. Los alumnos son inducidos a privilegiar la especialización, es decir, la adquisición de competencias y habilidades mejor valoradas por el mercado laboral, por sobre la visión de conjunto que ofrecen las grandes teorías económicas. Otra barrera institucional proviene de organismos multilaterales y la oficialidad del Estado; es el caso de las evaluaciones de programas y proyectos públicos 
donde se privilegia el uso de metodologías ortodoxas ${ }^{3}$.

Desde otra perspectiva, el acercamiento de los historiadores del pensamiento económico a la comunidad de historiadores y, en consecuencia, su alejamiento de la de los economistas, se refuerza por una exigencia metodológica, la tendencia a mejorar los estándares historiográficos de la producción en HPE. En tal sentido, Roy Weintraub (1996), al preguntarse sobre las características de una contribución legítima a la HPE, planteó la necesidad de exigir un estilo de erudición que es común entre historiadores, pero escaso entre los economistas. Esto es, entre otros, el uso de fuentes de información tanto primarias como secundarias, evidencia circunstancial, conocimientos del contexto y la exposición de los datos a la crítica tanto interna como externa, con el objeto de tener el máximo grado de objetividad posible. Weinstraub (1996) concluye que un "buen" economista no es necesariamente un "buen" historiador del pensamiento económico y viceversa.

Esta exigencia de dar buen uso al conjunto de técnicas y teorías relacionadas con el estudio, el análisis y la manera de interpretar la historia, como condición necesaria para el desarrollo de la investigación en HPE, implica un reto metodológico, un costo de entrada alto para el economista que pretende incursionar en este campo de estudio.

Por otro lado, el desconocimiento del rigor metodológico de la disciplina histórica, por parte de los economistas, con frecuencia se oculta tras el prejuicio de exaltar los métodos, las técnicas y las herramientas cuantitativas, al

3 La instauración de los modelos de la vertiente neoclásica ha sido promovida por los principales centros de investigación y de poder, tales como el Banco Mundial, el FMI, y los principales centros académicos anglosajones. Lo anterior se ha implantado o ha sido seguido por los diferentes gobiernos de países en vías de desarrollo y centros de investigación de diferentes naciones, sobremanera las que reciben ayudas condicionadas o préstamos de los principales organismos multilaterales. tiempo que se menosprecian los métodos procedentes de otras ciencias sociales. Al respecto, Gómez y Tobón (2009, p. 247) han señalado que la palabra "historia" produce dos efectos entre los economistas: miedo y vergüenza. Miedo porque usualmente los economistas no tienen una formación rigurosa en los métodos de trabajo de los historiadores. Vergüenza, porque provienen de un cuerpo de conocimiento que pretende ser una "ciencia"; luego el uso de métodos históricos puede interpretarse como una salida fácil que oculta una incapacidad en el uso de herramientas matemáticas o estadísticas. La arrogancia del conocimiento atenta contra el diálogo de saberes.

La pérdida de interés por la HPE, en la formación y práctica de los economistas, plantea la necesidad de justificar el objeto del campo de estudio y su pertinencia dentro de la economía. En lo anterior, está implícita la cuestión de qué tipo de HPE se debe promover, es decir, señalar posibles caminos o rutas a seguir. En este sentido, se propone reivindicar la importancia de investigar los fundamentos conceptuales de las diferentes teorías. Como condición necesaria en la práctica de la HPE, los conceptos definen y articulan los bloques constructivos esenciales de la teoría, luego su estudio es relevante en el desarrollo de la ciencia económica.

Los economistas necesitan estudiar y practicar la HPE. El encuentro con los grandes autores de la teoría económica implica reconocer la pluralidad de voces que participan en la construcción del campo de estudio, los consensos y disensos en torno a la definición de la disciplina, la diversidad de contextos y de identificación e interpretación de los problemas, de las distintas visiones sobre el funcionamiento de los sistemas económicos y de la variedad de teorías y modelos que de ella se derivan. Así, una visión retrospectiva de la economía permite entender la trayectoria de evolución, los desarrollos y cambios, de la disciplina, un camino que no es lineal, que parece más bien, como señala Roncaglia (2006), un ovillo de lana enredada.

Para desenredar el ovillo es preciso, primero, identificar los hilos conductores, los elementos transversales que mantienen la cohesión del 
campo de estudio, que, en el caso de la economía, como ya se señaló, son las grandes preguntas; y, segundo, concentrarse en los fundamentos conceptuales de las diferentes teorías. Para entender la forma en que se desenvuelve el hilo es necesario considerar los conceptos como unidades de análisis, teniendo en cuenta dos consideraciones básicas; la primera, que el significado de un concepto puede cambiar cuando se transita de un autor a otro o de una teoría a otra y, la segunda, que los cambios en la estructura analítica están relacionados con cambios en los fundamentos conceptuales (Roncaglia, 2006, p. 9). El cambio conceptual es así tanto indicador como agente en la evolución de la teoría económica.

La exactitud filológica claramente es un elemento básico para juzgar la validez científica de las ideas de un autor, la reconstrucción de la estructura lógica de su pensamiento no puede obviar, entonces, la fase de conceptualización (Schumpeter, 1954/1994, p. 79). Por esta razón, los métodos de la historia de los conceptos, aplicados a la HPE, proporcionan sentido y coherencia a la evolución de la teoría económica y son relevantes para el estudio tanto de teorías generales como de los campos específicos. De esta manera, la HPE no se circunscribe al estudio de las teorías generales y al desarrollo de modelos referenciales para las distintas subdisciplinas (Gómez y Tobón, 2009, pp. 246-247), sino que la misma metodología aplica a las diferentes especialidades. Es decir, se propone que la relación entre subdisciplinas, además de la referencia a las grandes preguntas y a alguna teoría general, también está presente en el lenguaje con que se comunican los economistas, en particular en sus piezas constructivas básicas: los conceptos. El lenguaje brinda un carácter general a la HPE, pues articula los problemas fundamentales con las teorías y los modelos, comunica a las distintas subdisciplinas entre sí y contribuye al trabajo de frontera en la teoría económica.

De esta manera, la HPE previene el riesgo de balcanización de la economía contemporánea, pues ofrece las herramientas de comunicación que permiten integrar los distintos compartimentos en que se ha dividido el campo de estu- dio, permite traducir y expresar en un lenguaje común los mensajes, teorías y modelos particulares, que cada isla emite en su propio dialecto. La función traductora de la HPE permite integrar los fragmentos y preservar el sentido de unidad y coherencia de la teoría económica, permite establecer la relación entre un dominio particular de las teorías especializadas y el codominio de las preguntas fundamentales y las grandes teorías de la ciencia económica. En la traducción, se establece el rango de cada especialidad, se identifican continuidades y rupturas y se reconoce el carácter complejo y cambiante del campo de estudio.

Así, la HPE nos ofrece una visión de conjunto del vasto campo de la teoría económica, lo que de por sí es importante a nivel didáctico y, ante todo, proporciona un "sentido de dirección" a la investigación económica:

\begin{abstract}
Es un ingrediente esencial tanto del debate teórico entre enfoques alternativos -dado que contribuye a aclarar las diferencias y modificaciones en sus representaciones del mundo- como del trabajo teórico dentro de cada enfoque, puesto que contribuye al desarrollo de los fundamentos conceptuales (Roncaglia, 2006, p. 33).
\end{abstract}

Adicionalmente, la HPE constituye una reserva de sentido y de sensatez para la teoría económica y para los economistas y, por lo tanto, para la interpretación del mundo de los fenómenos económicos:

La historia del pensamiento económico es una reserva de sentido en la medida en que nos permite tener una visión más integral del desarrollo de la economía, permitiéndole a la disciplina pensarse a sí misma en la continuidad de unas grandes preguntas. Es una reserva de sensatez, en cuanto permite mantener en perspectiva los resultados que parecen descubrimientos modernos pero que, en realidad, tienen antecedentes y desarrollos alternativos en el presente extendido de la disciplina (Álvarez y Hurtado, 2010, p. 279).

La economía es una ciencia social. Incluso reducidos a su ámbito estrictamente mercantil, los procesos de producción, intercambio y consu- 
mo son fenómenos sociales. De allí su carácter complejo y la necesidad de identificar la economía dentro del conjunto más amplio de las ciencias sociales. La HPE tiene la tarea de identificar el lugar de la economía dentro de las ciencias sociales, establecer canales de comunicación y promover el trabajo interdisciplinar en campos como la antropología, la historia y la sociología económicas. Esta función permite reconocer la interdependencia de la economía con otras disciplinas y de las ideas y los hechos económicos con sus respectivos contextos. Elementos esenciales para entender la interacción social. La HPE también permite reflexionar sobre el cómo y el por qué los economistas piensan y hacen su disciplina. Es decir, la reflexión metodológica y epistemológica; todo lo cual incluye los criterios de validez y alcance establecidos por la comunidad académica sobre temas, preguntas, metodologías y teorías.

Se ha señalado que la HPE es necesaria en la enseñanza y la práctica de la economía. Ahora es necesario insistir en que el historiador del pensamiento económico no debe renunciar a considerarse economista. Es decir, a contribuir con su trabajo al avance de la teoría económica y la producción de nuevas ideas.

Por su naturaleza, la HPE explora en el pasado en la búsqueda de ideas y de bloques constructivos potencialmente útiles, en la medida en que puedan ser conscientemente reinterpretados o traducidos en un lenguaje moderno, para el desarrollo de la teoría económica o para la construcción de teorías económicas alternativas. Para esto se requiere, en primera instancia, restaurar el significado original de las teorías, a la luz del contexto, de los usos y los criterios de verdad contemporáneos al autor. Es decir, en la lectura de un autor del pasado, la interpretación nos exige encontrar su propia voz ${ }^{4}$.

4 Conviene aclarar que, si bien la ciencia positivista se plantea como reto ofrecer una descripción exacta de la realidad objeto de estudio, en la HPE se reconoce que tal reto es tan solo una pretensión, a la que, por supuesto, no se debe renunciar. Así lo constata la profusión de artículos y libros dedicados a cuestiones de interpretación y reinterpretación de la obra de los grandes autores.
Luego de lo cual, es posible traducir las ideas a un lenguaje moderno para ver qué nos pueden decir sobre nuestro presente.

La HPE presenta dos características básicas. Primero, privilegia el estudio e interpretación de la obra de los grandes autores, principalmente de Smith, Ricardo, Marx, Walras y Keynes; y, segundo, metodológicamente ha evidenciado su predilección por la exégesis textual ${ }^{5}$. El énfasis en los grandes autores se debe a la originalidad e importancia explicativa de sus contribuciones a la teoría económica y se refuerza por la disponibilidad de ediciones críticas de sus obras, la publicación de sus biografías y correspondencia, lo que en ocasiones induce a descuidar la búsqueda de nuevas fuentes primarias, tanto de los grandes autores como de otros considerados de menor importancia. La inclinación por el enfoque de la exégesis textual y la pretensión de extraer el significado original de los textos implica dejar de lado el contexto en el cual fueron enunciadas las teorías.

\section{El método de la matriz conceptual}

Peterson y Bean (1998) proponen el uso de una matriz conceptual como dispositivo para organizar los cursos de HPE. El propósito del método es ayudar a los estudiantes a mejorar la comprensión de la HPE y, en el proceso, promover el desarrollo de habilidades cognitivas de alto nivel, un pensamiento más elevado en términos de complejidad y una actitud más crítica para comparar y evaluar las distintas teorías económicas. Los autores toman como referencia el esquema de desarrollo cognitivo por niveles presentado por Thoma (1993), según el cual el pensamiento crítico se define

5 Maria Cristina Marcuzzo (2008, pp. 108-113) clasifica en cuatro los estilos o métodos de investigación en la historia del pensamiento económico. Esto a partir de la naturaleza de las "técnicas" involucradas en la disciplina: a) exégesis textual; b) "reconstrucciones racionales"; c) "análisis contextual"; y d) "narrativa histórica". 
como la capacidad para identificar aspectos relevantes de un tema y utilizar los métodos apropiados y las evaluaciones estándar para alcanzar una conclusión. En cuanto al desarroIlo cognitivo, se plantean cuatro niveles: dualismo, multiplicidad, relativismo contextual y decisiones adecuadas al contexto. En el primer nivel, el estudiante cree que existen verdades absolutas; luego reconoce la diversidad de puntos de vista, es decir, advierte que en el conocimiento hay elementos de incertidumbre y subjetividad. Posteriormente, emplea criterios disciplinarios para valorar los distintos puntos de vista y; al llegar al cuarto nivel, reconoce que cada disciplina tiene sus propios criterios, que estos se complementan, y que en las decisiones que se toman en la "vida real" están mezclados los criterios disciplinares con sistemas de creencias y valores personales.

El dispositivo propuesto consiste en un cuadro o matriz de doble entrada que le ayuda al estudiante a identificar, organizar, registrar, sistematizar y criticar los contenidos del curso (ver Tabla 1). Las columnas corresponden a autores o escuelas de pensamiento económico; son unidades temáticas del curso, que Peterson y Bean (1998) sugieren definir en correspondencia con el libro de texto utilizado como material de guía para el desarrollo del curso. Los autores y escuelas que ellos proponen son, en su orden de presentación: economía neoclási- ca, griegos, escolásticos, mercantilismo, fisiocracia, Smith, Malthus-Say, Ricardo, J. S. Mill, Marx, Marginalismo, Marshall-microeconomía neoclásica, macroeconomía neoclásica, Institucionalismo y Keynes.

Las filas de la matriz corresponden a temas específicos o problemas recurrentes en la HPE, desarrollados por la mayoría de los autores o escuelas, los cuales están agrupados en tres grandes secciones: aspectos normativos, contexto histórico y temas económicos. La primera sección incluye: naturaleza de la riqueza, naturaleza de la persona humana, concepción de justicia y papel del gobierno. La sección de contexto histórico hace referencia al escenario en que se desarrollaron las teorías; y la sección de temas económicos se integra por: teoría de precios, mercado, división del trabajo, comercio internacional, leyes de granos o precio de los alimentos, teoría monetaria, distribución, tamaño y crecimiento de la población y preocupaciones macroeconómicas. En la propuesta de los autores, la mayoría de las actividades del curso (clases, discusiones, grupos de estudio, exámenes, trabajos escritos y presentaciones orales) se orientan al desarrollo de la matriz. Cada unidad temática del curso se trabaja en una columna y se registra en las celdas la información correspondiente a cada uno de los temas establecidos.

\begin{tabular}{|c|c|c|c|c|}
\hline & & Síntesis neoclásica & Grecia & Escolástica \\
\hline $\begin{array}{l}\text { Cuestiones } \\
\text { normativas }\end{array}$ & $\begin{array}{l}\text { La naturaleza de la riqueza } \\
\text { La naturaleza de la persona humana } \\
\text { Concepción de la justicia } \\
\text { Papel del gobierno }\end{array}$ & & & \\
\hline Contexto histórico & Escenario & & & \\
\hline $\begin{array}{l}\text { Cuestiones } \\
\text { económicas }\end{array}$ & $\begin{array}{l}\text { Teoría de precios } \\
\text { Mercado de trabajo / División del trabajo } \\
\text { Comercio internacional } \\
\text { Leyes de granos / Precio de los alimentos } \\
\text { Teoría monetaria } \\
\text { Distribución } \\
\text { Tamaño de la población / Crecimiento } \\
\text { Preocupaciones macroeconómicas }\end{array}$ & & & \\
\hline
\end{tabular}

Nota: Las columnas no desplegadas son: Mercantilismo, Fisiocracia, Smith, Malthus/Say, Ricardo, J. S. Mill, Marx, Marginalismo, Marshall/Microeconomía neoclásica, Macroeconomía neoclásica, Institucionalismo, Keynes. 
Peterson y Bean (1998, pp. 265-267) destacan tres beneficios del método de la matriz: 1) el curso se enfoca en los temas clave y sus significados, permite identificar los datos significativos, los temas y problemas que organizan la disciplina, y descartar información irrelevante; 2) la matriz es un vehículo por medio del cual se clasifican ideas, proporciona un marco para procesar información. Al establecer categorías significativas, permite conectar datos a una estructura de conceptos y facilitar el proceso de transferencia de información del corto plazo a la memoria de largo plazo y establecer marcos o esquemas interpretativos; y 3) el proceso de completar las celdas de la matriz, columna tras columna, promueve el desarrollo de pensamiento crítico. En el desarrollo de la matriz, los estudiantes enfrentan cuatro etapas o niveles del crecimiento cognitivo: en un primer nivel, la comprensión lectora y las clases exigen identificar y relacionar a los autores con sus respectivas ideas o teorías, el acento se coloca en las palabras clave y sus significados; en un segundo nivel, los estudiantes se enfrentan a la apreciación de múltiples puntos de vista, comparan las ideas de una escuela nueva respecto a las previamente examinadas, identifican semejanzas y diferencias, consensos y controversias, los múltiples puntos de vista de los autores o escuelas respecto a temas específicos, lo que permite reconocer el diálogo entre distintos enfoques; en el tercer nivel, se desarrolla la habilidad para emplear criterios diversos y evaluar ideas en competencia, se establecen relaciones entre ideas o teorías, se evalúa si las teorías son lógicamente consistentes y se establecen relaciones entre las ideas de cada autor o escuela y su correspondiente contexto histórico; en un cuarto nivel, el más alto, el estudiante se enfrenta a la responsabilidad de hacer elecciones, de establecer valores personales y criterios disciplinarios. En el caso de los valores, el estudiante debe identificar cómo la posición de los economistas acerca de preguntas económicas clave puede estar influenciada por sus creencias y valores subyacentes.

Peterson y Bean (1998) reconocen dos reservas básicas con respecto a la herramienta metodológica que proponen. La primera reserva consiste en la posible falsa impresión de uni- formidad de la matriz. La segunda remite al debate entre absolutistas y relativistas. Respecto a la primera reserva, la impresión de uniformidad y consistencia del pensamiento de individuos y de escuelas, resultante de la ordenación de información en la matriz, oculta las inconsistencias, divisiones, desacuerdos y conflictos que con frecuencia se encuentran en la HPE, incluso al interior de una misma escuela o autor. Evidentemente, las grandes obras contienen supuestos y afirmaciones conflictivas. Con el objeto de contrarrestar esta falsa apariencia de pulcritud, corresponde al profesor explicar a los estudiantes cómo la selección de escuelas de pensamiento y de autores individuales, si bien tiene por objeto encontrar patrones comunes, no desconoce la riqueza y variedad de preguntas, métodos, opiniones y visiones de distintos autores al interior de una misma escuela. Igualmente, resaltar los disentimientos o diferencias de énfasis al interior de las escuelas de pensamiento, así como indicar que el ejercicio de la matriz conceptual es tan solo un marco inicial que siempre podrá ser enriquecido con otros autores y escuelas, para establecer nuevos diálogos y debates. Conviene insistir en lo obvio: la matriz conceptual es un medio, una herramienta, no un fin en sí misma.

La propuesta de iniciar el desarrollo del curso, y en consecuencia de la matriz, con la presentación de la economía neoclásica moderna, remite inevitablemente al debate entre absolutistas y relativistas. Los autores sostienen que la matriz trasciende esta controversia, pues utiliza los dos enfoques; al proponer como punto de partida la presentación de la economía neoclásica, la visión se torna absolutista, el paradigma neoclásico se constituye en referente de comparación implícito frente a las demás escuelas de pensamiento. De esta manera, se induce al estudiante a pensar en cómo el pensamiento económico progresó hasta la forma que predomina en la actualidad. Los autores proponen que este sesgo se contrarresta con la presencia, en una de las filas de la matriz, de una sección de "contexto histórico", lo cual sugiere un enfoque relativista que busca ponderar las teorías en sus propios términos, al conectar el análisis económico con factores sociales, políticos y culturales más amplios (Peterson y Bean, 1998, pp. 271-272). 
En conclusión, la matriz conceptual puede ser una herramienta útil para conectar temas de la HPE que usualmente se presentan como desconectados. La precisión en la definición de los conceptos, históricamente determinados, permite destacar la importancia de las condiciones y presupuestos sobre los cuales han sido construidas las distintas teorías y la interrelación entre estas. La matriz, en la medida en que le permite al estudiante ponderar la diversidad de opiniones y emplear una variedad de criterios para evaluar las distintas perspectivas teóricas que compiten entre sí, tiene el potencial de promover en los estudiantes un pensamiento de orden superior. La matriz permite sistematizar información y producir un cuerpo de conocimiento potencialmente interconectado alrededor de cuestiones significativas.

La nueva historia intelectual, a partir de la investigación sobre el uso del lenguaje en contextos históricos, proporciona herramientas metodológicas que pueden potenciar la capacidad del método de la matriz para promover las capacidades cognitivas de los estudiantes y lograr un pensamiento complejo y crítico. El recurso a la semántica histórica tiene que ver con el análisis de los actos de habla, sus contextos y las intenciones e interpretaciones de los hablantes. Koselleck (2004) señala que "los conceptos permiten guardar y retener las experiencias incluso cuando éstas ya se han desvanecido" (p. 24). Los conceptos son creados, establecidos y modificados a través de la comunicación, sometidos a variaciones constantes, generalmente sutiles, casi imperceptibles, pero en ocasiones abruptas y profundas. De esta manera, cualquier período puede estar bajo escrutinio, aunque naturalmente las fases de cambio radical suelen ser las más interesantes desde el punto de vista histórico. Por esta razón los conceptos no se pueden considerar como unidades de análisis preestablecidas: su naturaleza es cambiante $y$, en consecuencia, la propuesta de matriz conceptual requiere flexibilidad, estar abierta a la posibilidad de incorporación de nuevos conceptos.

Por lo general, el cambio conceptual sigue al cambio social, en sus distintas dimensiones: políticas, económicas, culturales o intelectua- les. Así, las variaciones en el contenido semántico de un concepto, o incluso la aparición de un concepto nuevo, son indicadores del cambio social. También ocurre que los conceptos son agentes del cambio, tienen una naturaleza performativa, como señala Richter (1987): "Los conceptos tanto registran como causan las transformaciones de las estructuras gubernamentales, sociales y económicas" (p. 252). Dada la interacción dinámica entre cambios sociales y conceptuales, tanto la historia de los hechos como la del pensamiento son necesarias para detectar y analizar las continuidades, modificaciones e innovaciones de los discursos económicos. Los análisis diacrónicos y sincrónicos facilitan identificar tanto similitudes y consensos como diferencias y contradicciones, al interior de una escuela o de un autor o entre escuelas o autores, y así mejora la comprensión de la forma en que evoluciona la teoría económica. La nueva historia intelectual, al cuestionar a la historia de las ideas, ofrece un conjunto de advertencias metodológicas básicas aplicables a la historia del pensamiento económico.

\section{El uso de la matriz conceptual}

Ekelund y Hébert (1998) plantean críticas adicionales, tanto generales como específicas, al método de la matriz. Entre ellas, se destacan dos reservas frente a la capacidad del método para generar pensamiento crítico y una mejor comprensión de la HPE. Por un lado, la posibilidad de que el método de la matriz pueda promover un pensamiento memorístico. Por otro lado, que el método no pueda cumplir con la expectativa de promover formas de pensamiento de más alto nivel, es decir, pensamiento crítico y complejo, pues en la práctica la matriz canaliza el pensamiento de los estudiantes en una vía predeterminada alrededor de conceptos preestablecidos, y de esta manera cierra la válvula del proceso de descubrimiento. Para estos autores, la matriz se convierte en una venda intelectual que condiciona a los estudiantes a leer la HPE con el único objetivo de rellenar espacios en una matriz, algo similar a llenar un crucigrama, perdiendo así los incenti- 
vos para encontrar, por sí mismos, ilustración en los textos primarios.

A partir de la experiencia en la utilización de la matriz conceptual ${ }^{6}, y$ con base en las reservas planteadas por Peterson y Bean (1998) y las críticas de Ekelund y Hébert (1998), a continuación se defiende el uso de la matriz conceptual. Se propone complementar el método de la matriz con las advertencias metodológicas procedentes de la nueva historia intelectual, como alternativa valiosa en la tarea de mejorar la comprensión de la HPE y en la promoción de habilidades cognitivas de alto nivel. Se complementa para destacar un pensamiento más elevado en términos de complejidad y una actitud más crítica para comparar y evaluar las distintas teorías económicas. Se destaca como resultado del reencuentro de la HPE con la historia de los hechos económicos y su potencial contribución al desarrollo de la investigación en teoría económica.

Los cursos de historia del pensamiento económico son un recurso escaso dentro de los planes de estudio de economía. En el caso colombiano es común que en los programas de economía se imparta un solo curso con carácter obligatorio, usualmente ubicado en los primeros semestres, que en algunos casos se puede complementar con cursos electivos o seminarios, según las preferencias de los estudiantes (Castro y Raffo, 2016, pp. 165-166). En este escenario, el objetivo del curso es ofrecer un panorama general de la evolución de la teoría económica y de su método. Esta situación induce a un sesgo en la selección de la bibliografía en favor de los libros de texto y en contra del uso de fuentes primarias. En la práctica, la elección, cualquiera que ella sea, tiene implicaciones importantes. En el caso de utilizar un texto guía para el desarrollo del

6 El autor del presente trabajo ha aplicado el método de la matriz en cursos de historia del pensamiento económico en universidades colombianas, en la Pontificia Universidad Javeriana y en el Colegio Mayor de Nuestra Señora del Rosario. En ambos casos, la asignatura se encuentra en el primer semestre del plan de estudios del programa de pregrado en Economía. curso, el éxito en la utilización del método de la matriz depende de la pulcritud, prolijidad o meticulosidad del texto guía. En tal sentido, el riesgo consiste en que la matriz reproduzca los sesgos de selección temática, de autores y escuelas, así como de interpretación por parte del autor del manual utilizado, algo inevitable dada la magnitud de la tarea a que se enfrentan los autores de los manuales. Por ejemplo, Ekelund y Hébert (1992) señalan en su libro de texto que se vieron obligados a "juzgar y elegir" las que para ellos son las principales ideas teóricas y metodológicas a partir de su propia "preferencia metodológica" y su "criterio informado" (pp. xvii-xviii). El libro de texto inevitablemente canaliza el pensamiento por una vía predeterminada, algo que paradójicamente estos autores critican de la propuesta de la matriz.

En caso de optar por la lectura de las fuentes primarias, el riesgo se traslada a la rareza, complejidad y extensión de las lecturas. En general, los estudiantes de primeros semestres que se enfrentan a este ejercicio declaran que las fuentes primarias están escritas con un lenguaje anticuado, extraño a las formas modernas, que los autores exhiben una fundamentación filosófica y cultural que les resulta ajena, y que, en ocasiones, esto dificulta la posibilidad de apreciar los significados que el autor pretende expresar. También les resulta difícil apreciar las conversaciones que el autor mantiene con sus interlocutores contemporáneos. Si a esto le sumamos la vastedad de las fuentes primarias, optar por esta alternativa hace irrealizable el objetivo de ofrecer al estudiante, en un semestre, un panorama relativamente completo de la HPE. En este punto, lo que se propone es combinar el uso de un texto guía, que brinde la visión de conjunto de la evolución del pensamiento económico, con la lectura de fragmentos de fuentes primarias que permitan, al menos parcialmente, apreciar los detalles, conocer a los protagonistas de la historia en su riqueza y complejidad analítica, apreciar su estilo, ubicarlos en su contexto e interrogar su pensamiento.

La presentación de la matriz en la primera semana de clases es recibida con curiosidad, 
entusiasmo y expectativa por parte de los estudiantes, que la asumen como un desafío intelectual. También es evidente que, al definirse en la matriz un conjunto de temas específicos, los estudiantes cuentan con una guía de lectura que los orienta para concentrarse en la búsqueda, identificación, clasificación, registro y análisis de información específica; y contar con información concreta les permite participar activamente en la clase. La exposición del profesor y las lecturas complementarias se orientan a cubrir temas no tratados en el libro de texto y ayudar a los estudiantes a entender los conceptos y establecer conexiones que resultan confusas o difusas durante la discusión. Si un autor o escuela de pensamiento no trata un tema particular, la celda se deja en blanco o, si es posible, se registra una razón significativa que justifique el no tratamiento del tema por parte del autor.

Al finalizar cada unidad temática del curso, es decir, el análisis de un autor o escuela particular, el trabajo de clase se enfoca en establecer las conexiones entre celdas y comparar con las columnas previamente diligenciadas. Esto tiene efectos cognitivos significativos en la medida en que el conocimiento nuevo, registrado en una nueva columna, se contrasta de manera natural con el conocimiento previo, las columnas anteriores, lo que favorece la transmisión de información de la memoria de corto plazo a la de largo plazo. En otras palabras, hace significativo el aprendizaje y facilita su retención. Siguiendo la estrategia propuesta por Peterson y Bean (1998, p. 263) se utilizan preguntas orientadoras como vehículo para promover el desarrollo de un pensamiento comparativo y crítico. La posibilidad de buscar conexiones entre celdas de una misma columna permite pasar de un nivel casi que enciclopédico, en el que se pone a prueba la comprensión de lectura y se maniobra con el significado de ideas aisladas en celdas, a un nivel en el que se conectan esas ideas a través de esquemas interpretativos que aproximan al estudiante a reconocer la complejidad del pensamiento de diversos autores y empezar a valorar la consistencia interna de sus aportaciones. Así como su dimensión pragmática, el contraste de esas ideas con un contexto histórico concreto en el que fueron enunciadas y sobre el que poten- cialmente intervino el autor.

El avance y contraste de celdas, a través de una fila, permite a los estudiantes identificar la variación en los contextos históricos, las permanencias y los cambios, los acuerdos y disputas en torno al contenido de cada uno de los conceptos establecidos. Allí el estudiante tiene la oportunidad de apreciar múltiples puntos de vista en competencia, relacionar ideas de distintos autores, reconocer la naturaleza de la evolución del conocimiento económico, con sus continuidades y rupturas, reconocer que las "leyes" de la economía no son inmutables. En general, los estudiantes identifican la importancia del relativismo histórico, lo que constituye una de las premisas centrales de la nueva historia política, "que todo conocimiento y significado se hallan ligados al tiempo y a la cultura" (Vallespín, 1995, p. 290). En particular, la matriz conceptual permite evidenciar "que cada palabra puede tener una multiplicidad de significados que se van adecuando a la realidad mudable" (Koselleck, 2004, p. 30).

En el contexto colombiano, es común que los cursos de HPE tengan dentro de sus propósitos formativos el desarrollo de competencias generales en los procesos de escritura. El método de la matriz es compatible con este objetivo; de hecho, son complementarios. La matriz permite la sistematización de información comparable, sentido por el cual constituye un insumo valioso que facilita el desarrollo de proyectos de escritura alrededor de temas problemáticos muy variados. Dentro de las posibilidades, se encuentra reconstruir los argumentos de un autor y evaluar su consistencia lógica, presentar distintos puntos de vista respecto a un tema y establecer criterios de evaluación para juzgar la validez relativa de un enfoque con respecto a otro. También permite establecer la relación existente entre un discurso económico y su contexto histórico, identificar presupuestos analíticos implícitos y juicios normativos. Así, el desarrollo de proyectos de escritura a partir de la matriz es una oportunidad para materializar la capacidad de los estudiantes para analizar un tema, identificar argumentos y contraargumentos, evaluar su consistencia utilizando criterios estándar, asumir una posición, defenderla y 
comprender sus implicaciones. Todas estas son operaciones mentales complejas.

Tanto en el desarrollo de la matriz como en los proyectos de escritura es probable que los estudiantes se sientan incómodos al identificar y tratar de analizar formas de pensamiento diversas. Para evitar que el proceso resulte desalentador, o incluso frustrante, se requiere del acompañamiento continuo por parte del docente, en particular en la formulación de preguntas pertinentes.

En este sentido, Peterson y Bean (1998, pp. 269-271) proponen como alternativa el desarrollo de ejercicios cortos de escritura o de grupos de trabajo colaborativo, orientados a partir de la formulación continua de preguntas que avanzan paulatinamente en complejidad a medida que se diligencia la matriz. El ejercicio inicial que proponen se realiza al empezar cada unidad temática del curso, cada columna de la matriz. Acá se pide a los estudiantes que expresen por escrito qué problema o preocupación motivó al autor a escribir; luego, a medida que se desarrolla un tema, en cada celda se presenta la oportunidad de preguntar cuál es la posición del autor respecto a un tema específico. Una vez se completa una columna, se les pide que comparen una celda individual de la nueva columna con la celda correspondiente de una columna ya desarrollada. Seguidamente se pregunta por la consistencia interna de un autor o escuela y la relación de esta con su contexto histórico. Por último, se orientan las preguntas a la conexión entre ideas y preceptos normativos.

En cuanto al desarrollo general del curso, la matriz conceptual se constituye en una guía que facilita la lectura, aunque no está libre de riesgos, pues los estudiantes concentran su atención en los elementos necesarios para diligenciar la matriz y, en ese sentido, es probable que desestimen o pasen por alto temas y conceptos que, a pesar de su importancia, no se encuentran en la matriz. Una alternativa para minimizar este riesgo consiste en otorgar libertad a los estudiantes para incorporar columnas o filas en la matriz y otorgar incentivos para que en efecto lo hagan. Por ejemplo, pre- miando la originalidad en el desarrollo de la matriz y en los temas de los trabajos escritos. De esta manera, la válvula a los potenciales descubrimientos queda abierta. También se recomienda incentivar el uso de fuentes primarias y el contraste de las fuentes secundarias.

La valoración del método de la matriz por parte de los estudiantes suele ser altamente positiva. En particular, los estudiantes aprecian el rigor de las definiciones conceptuales y el ejercicio de síntesis implícito en el diligenciamiento de cada una de las celdas de la matriz. De la misma manera, valoran el ejercicio de realizar comparaciones entre escuelas de pensamiento económico a través de procesos de escritura.

\section{Algunas advertencias metodológicas}

Quentin Skinner (1969/2007) cuestiona la historia de las ideas. Esta estudia e interpreta el canon de textos clásicos como portadores de una sabiduría inmemorial, de ideas universales, de conceptos fundamentales y problemas perdurables, hace abstracción de las condiciones sociales y los contextos intelectuales en que surgieron. Desconocer el contexto del autor, la cultura en la que está inmerso, implica el riesgo de imponer las expectativas y los prejuicios propios de quien oficia como intérprete. Es un problema de configuración mental del observador: es inevitable que este interprete, organice y ajuste sus percepciones y pensamientos conforme a modelos preconcebidos que determinan lo que piensa y lo que percibe, como señala Skinner (1969/2007): "Debemos clasificar para entender, y sólo podemos clasificar lo que no nos es familiar en términos de lo que es familiar" (p. 65). Es decir, si el observador desconoce el contexto, su interpretación de la historia va a responder a un conjunto de criterios e intereses preconcebidos y se produce una suerte de profecía autocumplida en la que la narrativa histórica se organiza de acuerdo con una constelación de suposiciones absolutas, procedentes de un paradigma dominante. Historizar los conceptos previene este riesgo. 
Skinner (1969/2007) intenta descubrir hasta qué punto el estudio histórico actual del pensamiento se encuentra afectado por la aplicación inconsciente de paradigmas, cuya familiaridad para el historiador disfraza una falta de rigor metodológico que conduce a absurdos históricos y produce mitologías o ficciones, no historias. En general, la Escuela de Cambridge centra su atención en el vocabulario político, tiene el propósito de identificar la intención de los autores a través del uso público del lenguaje (Vallespín, 1995). Es decir, va más allá de la comprensión literal de los textos, de la exégesis textual, y privilegia la comprensión histórica de los mismos: el texto, en su nivel o dimensión locutiva, no es una unidad autónoma de sentido, su grado de validez depende del contexto histórico, de la dimensión ilocutiva ${ }^{7}$. De esta manera, para recuperar el sentido intencional es necesario reconstruir el contexto lingüístico en el que se produce o publica el texto. Reconstruir las convenciones que limitan las afirmaciones posibles. Skinner (1969/2007) propone cuatro tipos de mitologías: de las doctrinas, de la coherencia, la prolepsis y el provincianismo, las cuales es deseable tener presentes para evitar los anacronismos y llevar a buen recaudo el método de la matriz.

La mitología de las doctrinas consiste en asumir que un autor del pasado está enunciando una doctrina sobre los problemas que en el presente se consideran constitutivos de la materia, del paradigma dominante, lo cual consciente o inconscientemente altera la interpretación del observador. Lo anterior significa dar como evidente la existencia y sentido de las doctrinas en el pensamiento de autores del pasado, a partir de las expectativas del historiador o del comentarista moderno, cuando quizás la intención del autor era otra. En otras palabras, la falacia consiste en atribuir a un autor del pasado el rol de precursor de una doctrina y de unas ideas, que le interesan al comentarista moderno y que no hacían parte del plano de posibilidades discursivas del autor.

7 Elemento inspirado en John L. Austin, en particular su libro Cómo hacer cosas con palabras.
Esta mitología presenta dos formas: la primera consiste en "tomar equivocadamente algunas observaciones sueltas y casuales de uno de los teóricos clásicos por sus «doctrinas» sobre los temas que el historiador está ya preparado a encontrar" (Skinner, 1969/2007, pp. 71-72). Es decir, se atribuye a un autor del pasado el título de precursor de una idea o teoría, se le asigna a sus ideas un significado que quizás él no tenía la intención de expresar. En unos casos, el comentarista moderno toma observaciones sueltas o casuales de un autor y las amalgama, de tal suerte que concluye que allí hay un cuerpo de doctrina; en otros casos, simplemente se tergiversan sus argumentos para hacerlos coincidir con el molde preestablecido por el investigador. El peligro consiste en atribuir a un autor del pasado, a partir de semejanzas casuales en la terminología, ideas, opiniones, argumentos o significados sobre los cuales el propio autor no tenía intención de realizar alguna contribución (Skinner, 1969/2007, pp. 66-68). Por ejemplo, considerar a Locke como padre del liberalismo es un mito, porque el liberalismo solo aparece como doctrina a finales del siglo XVIII.

La segunda forma de esta mitología es inversa a la anterior. Consiste en considerar que un autor del pasado cometió una omisión o error al no producir una doctrina compatible con las expectativas o con el sistema de creencias del comentarista moderno. Este hecho constituye una forma de arrogancia del presente representado en pretender juzgar a un autor del pasado a partir de conocimientos que probablemente no estaban a su disposición. Por ejemplo, criticar a Smith por no profundizar en el análisis de la influencia de la maquinaria sobre los intereses de las diversas clases sociales; tema importante para Ricardo, pero que no representaba el mismo interés para los teóricos que escribieron antes de la Revolución Industrial.

Esta mitología se produce porque el paradigma moderno establece un conjunto de "verdades canónicas" que determinan la dirección de la investigación histórica. Es un caso de hipóstasis: se atribuye a una doctrina, una categoría abstracta, el estatus de entidad real, un tipo 
ideal de doctrina inmanente en la historia que espera a ser descubierta. Es el investigador quien desde el presente impone una doctrina y sus propios prejuicios al autor del pasado. Esta imposición limita las posibilidades de interpretación histórica de las contribuciones de los autores (Skinner, 1969/2007, pp. 72-73). Una forma prevaleciente de esta mitología, en particular dentro de la literatura exegética, consiste en criticar a los escritores clásicos por no haber realizado la contribución más sistemática que eran capaces de hacer a su disciplina. Se parte de presuponer que ellos tuvieron la intención de desarrollar de manera sistemática algún tema, y luego se les juzga por no haber sido capaces de desarrollar sistemática y totalmente ese tema. Sin saber si realmente tuvieron o pudieron haber tenido la intención de hacerlo (Skinner, 1969/2007, p. 73).

La mitología de la coherencia consiste en asumir una coherencia que no está presente en el texto o en el pensamiento de un autor, problema que se exacerba ante la dificultad de preservar el énfasis y el tono apropiados de una obra al parafrasearla, y en consecuencia la "tentación de encontrar un «mensaje» que se pueda abstraer y comunicar más fácilmente" (Skinner, 1969/2007, p. 76). El exégeta asume que su deber es descubrir la coherencia interna de las doctrinas, encontrar una frase reveladora, buscar una y otra vez, unir frases sueltas de los escritos, usar metáforas del esfuerzo y la búsqueda del autor, con la obsesión de mostrar la coherencia integral de su pensamiento, de su obra. "La ambición es siempre «llegar» a «una interpretación unificada» para «alcanzar» una «visión coherente del sistema del autor»" (Skinner, 1969/2007, p. 77). Se asume la coherencia, un único corpus de pensamiento en un autor. Tal puede ser el caso de la interpretación de las dos grandes obras de Adam Smith, asumir acríticamente la complementariedad de la Teoría de los Sentimientos Morales con la Riqueza de las Naciones. Si se asume en primer lugar que en sus escritos subyace una teoría coherente, por ejemplo, del orden social, entonces sus escritos convergen a un único cuerpo de pensamiento, necesariamente coherente ¿Por qué asumir un pensamiento único, una intención de coherencia en toda su obra? Al hacerlo, se corre el riesgo de forzar una respuesta que permita resolver las contradicciones del autor buscando una coherencia de la cual no tenemos certeza que buscara o aspirara a tener.

La ausencia de un sistema coherente de pensamiento se vuelve motivo de reproche. Por urgencia ideológica y conveniencia exegética, las diversas declaraciones de un autor de manera artificial son objeto de ordenación sistemática y los aportes se clasifican de acuerdo con el modelo al que se supone que aspiran, el que impone el investigador. La mitología de la coherencia se desarrolla en dos direcciones: la primera consiste en descartar las declaraciones de intención que los autores mismos realizan sobre lo que están haciendo, incluso rechazar obras completas que afectan la coherencia de su sistema de pensamiento. Locke, por ejemplo, en sus primeros escritos, pudo estar interesado en proponer y defender un sistema conservador, o incluso autoritario. Sin embargo, se le suele considerar como un teórico político "liberal". Esto por las opiniones que sostuvo cuando era adulto. La mitología se produce cuando el analista moderno asume que el autor ha evolucionado o reconsiderado su pensamiento, que repudia sus opiniones anteriores, en fin, el joven Locke no era todavía «Locke».

La segunda dirección consiste en suponer que los textos clásicos exhiben una "coherencia interna", una suerte de verdad revelada que impone al intérprete la tarea de quitar del camino cualquier obstáculo a su revelación. Así, cuando en el pensamiento de un autor se identifica una inconsistencia o una contradicción, el intérprete asume como propia la tarea de desvirtuarla, de tratar de demostrar que las contradicciones o antinomias son aparentes. Por ejemplo, no permitirle a Marx haber desarrollado y cambiado su pensamiento, desde los tintes humanistas de los manuscritos de economía y filosofía de 1844 hasta el sistema aparentemente mecanicista, esbozado dos décadas después, en el primer volumen de El Capital. Por ejemplo, unas veces se sugiere construir un análisis estructural en el que las divergencias se puedan entender como partes de un «cuerpo» de pensamiento; 
otras veces se informa que, al contrario, la existencia del material anterior demuestra que Marx estaba obsesionado con una visión moral de la realidad, y que se puede emplear para desacreditar sus posteriores pretensiones científicas (Skinner, 1969/2007, p. 81).

Las dos mitologías que se han discutido surgen del hecho de que, al examinar a un autor, el historiador del pensamiento está predispuesto por algún prejuicio sobre las características definitorias de la disciplina a la que pretende hacer una contribución.

Cuando el comentarista moderno desencaja los textos de su contexto de enunciación y coloca en ellos sus propias ideas y significados, se produce la mitología de la prolepsis. La prolepsis consiste en la búsqueda de un significado retrospectivo y no del significado histórico, el del autor en su época. Por ejemplo, considerar a Platón como un teórico del totalitarismo, un concepto del siglo XX, por sugerir que un consejo de sabios tendría mejor criterio que los ciudadanos para determinar lo que le conviene a la sociedad y que podría orientarla por medio de leyes. La significación histórica de una obra se confunde con la explicación de lo que el autor estaba proponiendo. El error está en pretender ver de manera inmediata los elementos "modernos" que el comentarista está programado para encontrar. En la prolepsis "el episodio ha de aguardar al futuro para comprender su significado" (Skinner, 1969/2007, p. 85), los argumentos están determinados por una forma teleológica, se pretende proporcionar una verdadera explicación del sentido de una acción del pasado y del interés que tiene para el comentarista actual. En la mitología de la prolepsis, el significado histórico de un episodio es desplazado por la significación que el observador moderno reclama. La posibilidad de que un observador pueda equivocarse al describir el significado que expresa un texto, es propia de cualquier intento de comprender una cultura ajena o un esquema conceptual poco familiar. ¿Cómo comunicar con éxito la comprensión de un texto del pasado dentro de la propia cultura del observador? El observador tiene que aplicar sus propios criterios familiares de clasificación y discriminación.
El provincianismo o localismo conceptual consiste en la tendencia a interpretar la obra de un autor del pasado a partir de una presunta influencia definida por el investigador en el presente. De esta manera, se postula una influencia absoluta del tipo Say ha sido influenciado por Smith, cuando quizás también fue influenciado por Turgot o Cantillon. El provincianismo se presenta de dos formas: la primera procede de cierta apariencia de las referencias, hablar erróneamente de la «influencia» de la obra de un autor anterior sobre la obra de un autor posterior, solo porque al investigador le parece identificar un argumento similar entre los dos autores. No se trata de que el concepto de influencia carezca de fuerza explicativa, el peligro se encuentra en que el concepto se utiliza de manera explicativa sin considerar las condiciones necesarias y suficientes para su aplicación. Las condiciones necesarias para poder invocar el concepto de influencia del tipo: $X$ ha sido influenciado por $Y$. Se requiere tener certeza sobre tres circunstancias: i) que $X$ estudió las obras de $Y$; ii) que $X$ no hubiese podido encontrar la doctrina relevante en ningún autor distinto a $Y$; y iii) que $X$ no hubiera llegado a la doctrina relevante de forma independiente.

La segunda forma de provincianismo se produce cuando el investigador intenta describir el sentido de una obra del pasado a partir de sus ideas presentes, asumiendo que estas son naturales, racionales y eternas. En este caso, una familiaridad engañosa puede llevar al investigador a tergiversar las teorías del pasado, riesgo que se corre, entre otros, cuando se utiliza un paradigma moderno para describir y comprender el pensamiento de los autores del pasado. Considérese, por ejemplo, cuando se trata de traducir la retórica de Smith a un lenguaje simplificado de diagramas y ecuaciones, proceso en el cual se corre el doble riesgo de diluir elementos que son ajenos a estas herramientas e incorporar anacronismos conceptuales en la explicación.

Estas advertencias metodológicas enriquecen el desarrollo de la matriz conceptual y las tareas de reflexión y escritura que de ella se pueden derivar. Con estas herramientas metodológicas básicas, el estudiante, orientado por el 
profesor, puede emprender una peregrinación al pasado para visitar a los grandes autores de la teoría económica e intentar establecer con ellos un diálogo respetuoso de los contextos de enunciación. Al eliminar las mitologías, tendrá la sensibilidad para identificar la novedad radical de sus ideas en un contexto histórico determinado y discernir hasta qué punto las ideas de un autor reflejan posiciones o valores normativos. Luego de esto, proceden las etapas de valoración de las teorías y de confrontación de distintos puntos de vista.

\section{Conclusiones}

En un contexto de creciente subdivisión de la ciencia económica en subdisciplinas, la HPE ofrece al menos dos elementos básicos para mantener la cohesión de un campo de estudio en crecimiento y expuesto al riesgo de disgregación. El primero de los anteriores es la referencia permanente a las grandes preguntas de la ciencia económica como elemento unificador. Allí tiene un lugar privilegiado la cuestión teórica de la emergencia del orden social, el funcionamiento del mercado, el sistema de precios, las instituciones y el cambio institucional. Por su parte, el segundo elemento constituye la fundamentación conceptual de la disciplina, la capacidad de la HPE para traducir a un lenguaje común los dialectos de cada subdisciplina, lo que por un lado permite reconocer la unidad y, por otro, representa una etapa ineludible del proceso de teorización. Dentro del proceso de teorización económica, la reconstrucción y análisis de la etapa de conceptualización permite reconocer que las teorías y los modelos están determinados históricamente y ofrece un sentido de orientación del progreso de la investigación teórica. Por lo anterior, los dos elementos justifican la importancia de la HPE en la formación de los economistas y demandan el desarrollo de capacidades cognitivas y pensamiento crítico.

Entonces, el reto de generar pensamiento crítico en economía, disciplina frecuentemente acusada de la promoción de un pensamiento único (el del paradigma neoclásico o mainstream), puede ser asumido por los historiadores del pensamiento económico. Lo anterior es plausible por cuanto la disciplina permite reconocer la naturaleza cambiante de los problemas como también la de las soluciones planteadas desde diversos puntos de vista. En general, la HPE es un campo privilegiado para la promoción de estrategias de enseñanza y aprendizaje crítico, que permiten el desarrollo de capacidades de análisis, síntesis, abstracción, argumentación y pensamiento divergente. Asimismo, la posibilidad de tales estrategias promueve la creatividad de los estudiantes mediante la búsqueda de múltiples alternativas de solución a los problemas, así como la identificación de presupuestos de carácter normativo implícitos en las ideas económicas.

Así las cosas, la propuesta de Peterson y Bean (1998) de usar una matriz conceptual para el desarrollo de cursos de HPE resulta valiosa, si bien presenta riesgos que deben sopesarse frente a sus ventajas. Los beneficios se asocian a la comprensión de las ideas de los autores a partir del examen crítico de sus definiciones y usos conceptuales.

Asimismo, el método propuesto invita al contraste permanente de ideas y permite identificar la complejidad de las cosmovisiones que subyacen en las diferentes teorías, al tiempo que se identifican sus potencialidades y límites. Por su parte, el desarrollo de capacidades cognitivas se pone a prueba de manera secuencial, por ejemplo, cada celda de la matriz reta al estudiante a buscar, identificar y clasificar información relevante; y cada columna lo invita a establecer relaciones e integrar las ideas en una estructura de análisis. Cuando se avanza a una nueva columna, surge la posibilidad de reconocer la novedad de las nuevas ideas por contraste con los conocimientos previamente adquiridos. Ello con la información registrada en columnas anteriores a la luz de los cambios en los contextos históricos. Frente a los riesgos, se señala que el uso de fuentes variadas, la combinación de fuentes primarias y secundarias, el desarrollo de trabajo colaborativo y los ejercicios de escritura son fundamentales para concretar el avance de los estudiantes y reducir los riesgos potenciales del método, tales como la promoción de un conocimiento 
memorístico y la orientación del aprendizaje de una manera predeterminada hacia un conjunto reducido de conceptos desarticulados.

Para evitar los riesgos mencionados resulta útil aproximarnos a las metodologías utilizadas para reconstruir la historia de los lenguajes políticos, en particular las mitologías propuestas por Q. Skinner (1969/2007), pues estas contribuyen a comprender las teorías económicas en sus propios términos. Sus advertencias metodológicas son necesarias para reconstruir rigurosamente la fundamentación del campo de estudio, la forma en que los autores del pasado representaron y establecieron relaciones con la realidad. En términos de la posibilidad de usar una matriz concep- tual para orientar un curso de HPE, la historia conceptual, y especialmente la advertencia del riesgo de crear mitologías, es necesaria para potenciar las ventajas de su implementación. De esta manera, el método de la matriz conceptual puede contribuir a promover un pensamiento crítico, que aumente los niveles de complejidad, que promueva la comparación y permita comprender la naturaleza evolutiva del pensamiento económico, así como establecer criterios disciplinares para la evaluación de las distintas teorías económicas. En una palabra, el método de la matriz es una alternativa valiosa. Para el particular, se subraya que este o cualquier otro método que contribuya a mejorar los procesos de enseñanza y aprendizaje de HPE debe ser celebrado.

\section{Referencias}

Álvarez, A. y Hurtado, J. (2010). Amenazas y ventajas de la enseñanza de la Historia del Pensamiento Económico. Lecturas de Economía, (73), 275-301. https://doi.org/10.17533/u0ea.le.n73a7874

Angrist, J. D. y Pischke, J. S. (2010). The Credibility Revolution in Empirical Economics: How Better Research Design Is Taking the Con out of Econometrics. Journal of Economic Perspectives, 24(2), 3-30. https://doi. org/10.1257/jep.24.2.3

Blaug, M. (2001). No History of Ideas, Please, We're Economists. Journal of Economic Perspectives, 15(1), 145-164. https://doi.org/10.1257/jep.15.1.145

Cardoso, J. L. (1995). Teaching the history of economic thought. The European Journal of the History of Economic Thought, 2(1), 197-214. https://doi.org/10.1080/10427719500000100

Castro, J. y Raffo, L. (2016). Algunas consideraciones sobre la estructura curricular de los programas de Economía en Colombia. Sociedad y economía, (30), 147-170. https://doi.org/10.25100/sye.v0i30.3904

Davis, J. B. (2019). Specialization, fragmentation, and pluralism in economics. The European Journal of the History of Economic Thought, 26(2), 271-293. https://doi.org/10.1080/09672567.2018.1555604

Ekelund, R. B. y Hébert, R. (1992). Historia de la Teoría Económica y de su Método (3 ed.). McGraw Hill.

Ekelund, R. B. y Hébert, R. F. (1998). Critical Thinking: Some Problems with the Matrix Method. The Journal of Economic Education, 29(3), 274-276. https://doi.org/10.2307/1183455

García, P. y Giraud, Y. (2016). The place of the History of Economic Thought in mainstream economics, 1991201 1, viewed through a bibliographic survey. Journal of the History of Economic Thought, 38(4), 431-462. https://doi.org/10.1017/S1053837216000481

Gómez, R. y Tobón, A. (2009). Reivindicando una definición para la historia del pensamiento económico. Lecturas de Economía, (71), 243-249. https://doi.org/10.17533/udea.le.n71 a4821

Goodwin, C. D. (2008). History of Economic Thought. En S. N. Durlauf y L. E. Blume (Eds.), The New Palgrave Dictionary of Economics (pp. 48-57). Palgrave Macmillan. https://doi.org/10.1057/978-1-349-951215_2624-1

Gordon, D. F. (1965). The role of the History of Economic Thought in the understanding of modern economic theory. The American Economic Review, 55(1), 119-127. https://www.jstor.org/stable/1816253 
Keynes, J. M. (1936/2013). The General Theory of Employment, Interest and Money (The Collected Writings of John Maynard Keynes Vol. VII). Cambridge University Press.

Koselleck, R. (2004). Historia de los conceptos y conceptos de historia. Ayer, 53(1), 27-45. http://revistaayer. com/articulo/711

Marcuzzo, M. C. (2008). Is history of economic thought a «serious» subject? Erasmus Journal for Philosophy and Economics, 1(1), 107-123. https://doi.org/10.23941/ejpe.v1i1.10

Peterson, D. y Bean, J. C. (1998). Using a Conceptual Matrix to Organize a Course in History of Economic Thought. The Journal of Economic Education, 29(3), 262-273. https://doi.org/10.2307/1 183454

Richter, M. (1987). Begriffsgeschichte and the History of Ideas. Journal of the History of Ideas, 48(2), 247263. https://doi.org/10.2307/2709557

Roncaglia, A. (2006). La Riqueza de las Ideas: Una Historia del Pensamiento Económico. Prensas Universitarias de Zaragoza.

Samuelson, P. A. (1987). Out of the Closet: A Program For the Whig History of Economic Science: Keynote Address at History of Economics Society Boston Meeting, June 20, 1987. Journal of the History of Economic Thought, 9(1), 51-60. https://doi.org/10.1017/S1042771600004002

Schumpeter, J. A. (1954/1994). Historia del Análisis Económico. Editorial Ariel.

Skinner, Q. (1969/2007). Significado y comprensión en la historia de las ideas. En E. Bocardo-Crespo (Ed.), El Giro Contextual: Cinco Ensayos de Quentin Skinner, y Seis Comentarios (pp. 63-108). Tecnos.

Thoma, G. A. (1993). The Perry framework and tactics for teaching critical thinking in economics. Journal of Economic Education, 24(2), 128-136. https://doi.org/10.2307/1183161

Trautwein, H. M. (2017). The last generalists. The European Journal of the History of Economic Thought, 24(6), 1134-1 166. https://doi.org/10.1080/09672567.2017.1378694

Vallespín, F. (1995). Giro lingüístico e Historia de las Ideas: Q. Skinner y la «Escuela de Cambridge». En R. R. Aramayo, J. Muguerza y A. Valdecantos (Comps.), El Individuo y la Historia: Antinomias de la Herencia Moderna (pp. 287-301). Paidós.

Weintraub, E. R. (1996). What Defines a Legitimate Contribution to the Subdiscipline "The History of Economics». HES Editorial. 